\title{
Determination of favorable blood glucose target range for stochastic TARgeted (STAR) glycemic control in Malaysia
}

\author{
Asma Abu-Samah ${ }^{1}$, Normy Norfiza Abdul Razak ${ }^{2}$, Ummu Kulthum Jamaludin ${ }^{3}$, \\ Fatanah Mohamad Suhaimi ${ }^{4}$, Azrina Mad Ralib ${ }^{5}$ \\ ${ }^{1}$ Institute of Energy Infrastructure, Universiti Tenaga Nasional Malaysia, Malaysia \\ ${ }^{2}$ Department of Electronics \& Communication Engineering, Universiti Tenaga Nasional Malaysia, Malaysia \\ ${ }^{3}$ Faculty of Mechanical Engineering, Universiti Malaysia Pahang, Malaysia \\ ${ }^{4}$ Department of Advanced Medical and Dental Institute, Universiti Sains Malaysia, Malaysia \\ ${ }^{5}$ Kulliyah of Medecine, International Islamic University Malaysia, Malaysia
}

\section{Article Info \\ Article history: \\ Received Dec 20, 2018 \\ Revised Feb 13, 2019 \\ Accepted Feb 27, 2019}

\section{Keywords:}

Blood glucose target range

Feedback control

Glycemic control

Intensive care units

Malaysian patients

\begin{abstract}
Stress-induced hyperglycemia is common in critically ill patients, but there is uncertainty about what constitutes an optimal blood glucose target range for glycemic control. Furthermore, to reduce the rate of hyperglycemic and hypoglycemic events, model-based glycemic control protocols have been introduced, such as the stochastic targeted (STAR) glycemic control protocol. This protocol has been used in the intensive care units of Christchurch and Gyulà Hospital since 2010, and in Malaysia since 2017. In this study, we analyzed the adaptability of the protocol and identified the blood glucose target range most favorable for use in the Malaysian population. Virtual simulation results are presented for two clinical cohorts: one receiving treatment by the STAR protocol itself and the other receiving intensive insulin therapy by the sliding scale method. Performance and safety were analyzed using five clinical target ranges, and best control was simulated at a target range of $6.0-10.0 \mathrm{mmol} / \mathrm{L}$. This target range had the best balance of performance, with the lowest risk of hypoglycemia and the lowest requirement for nursing interventions. The result is encouraging as the STAR protocol is suitable to provide better and safer glycemic control while using a target range that is already widely used in Malaysian intensive care units.
\end{abstract}

Copyright $(2019$ Institute of Advanced Engineering and Science. All rights reserved.

\section{Corresponding Author:}

Asma Abu-Samah,

Institute of Energy Infrastructure, Universiti Tenaga Nasional Malaysia,

Jalan IKRAM-UNITEN, 43000, Kajang, Malaysia.

Email: Asma@uniten.edu.my

\section{INTRODUCTION}

In 2001, a randomized controlled trial reported that significant reduction in mortality could be achieved through glycemic control compared with that using conventional insulin therapy [1]. Improved control was achieved with a lower and stricter blood glucose (BG) target range of 4.4-6.1 mmol/L (80-110 $\mathrm{mg} / \mathrm{dL})$ compared with that obtained with the conventional range of $<10.0-11.1 \mathrm{mmol} / \mathrm{L}(180-200 \mathrm{mg} / \mathrm{dL})$. These findings changed the standard of intensive care worldwide. However, subsequent randomized controlled trials targeting similar BG ranges have shown no benefits with respect to mortality, but a significantly increased risk of hypoglycemia [2-4]. In the NICE-SUGAR study, the largest multi-center study to date [4], a BG target of $<10.0 \mathrm{mmol} / \mathrm{L}$ effectively lowered mortality compared with that obtained with the strict target range of $4.5-6.0 \mathrm{mmol} / \mathrm{L}(81-108 \mathrm{mg} / \mathrm{dL})$. These benchmark results again changed clinical 
choice; however, this time, it was toward a wider and slightly higher target range [5], [6] that aimed to control hyperglycemia while reducing the risk of hypoglycemia.

Glycemic control requires frequent measurements of BG level as well as estimates of recommended insulin doses, which are based on an adopted protocol that heavily relies on a BG target range. To obtain glycemic control that is both better (less risk of hyperglycemia) and safer (less risk of hypoglycemia), computerized protocols have been recommended for use to provide the recommended insulin dose and BG measurement interval [7]. Although a growing number of intensive care units (ICUs) are starting to use validated computer algorithms with beneficial effects on glycemic control [e.g., EndoTool, LOGIC, GlucoSpace and stochastic targeted (STAR)] [8-13], such tools are not yet widely implemented in clinical practice. The clinically validated STAR glycemic control protocol uses model-based insulin sensitivity (SI) $[14,15]$ to characterize and forecast per-patient changes in metabolic state.

The STAR protocol is not only patient-specific but also flexible. This protocol has been used in the intensive care units of Christchurch and Gyulà Hospital since 2010, and in Malaysia since 2017 [16]. The clinical target ranges and maximum risk (BG $<4.0 / 4.4 \mathrm{mmol} / \mathrm{L}$ ) can be set by the clinical staff, and the model selects treatments that are justified by their predicted effects on the full range of possible BG outcomes. Its default target range is $4.4-8.0 \mathrm{mmol} / \mathrm{L}(80-145 \mathrm{mg} / \mathrm{dL})$, with a $5 \%$ risk of hypoglycemia. These values are based on the trial on the Christchurch and Gyulà cohort [17] and correspond to the best performance with their skewed distribution and patient demographics. Figure 1 presents the results of a data collection in which fellow Malaysian collaborators aimed to identify the default clinical target ranges implemented in Malaysian ICUs glycemic control. The figure shows the number of Malaysian hospitals ICU (both government and research universities) according to their BG target ranges used with the sliding scale method. In total, 24, 11, 4 , and 3 hospitals use target ranges of 6.0-10.0, 6.0-8.0, 4.0-6.0, and 8.0-10.0 mmol/L. Other target ranges are only used by single hospitals, based on expert judgment. Three of the four target ranges stated above require strict control within the range of $2.0 \mathrm{mmol} / \mathrm{L}$.

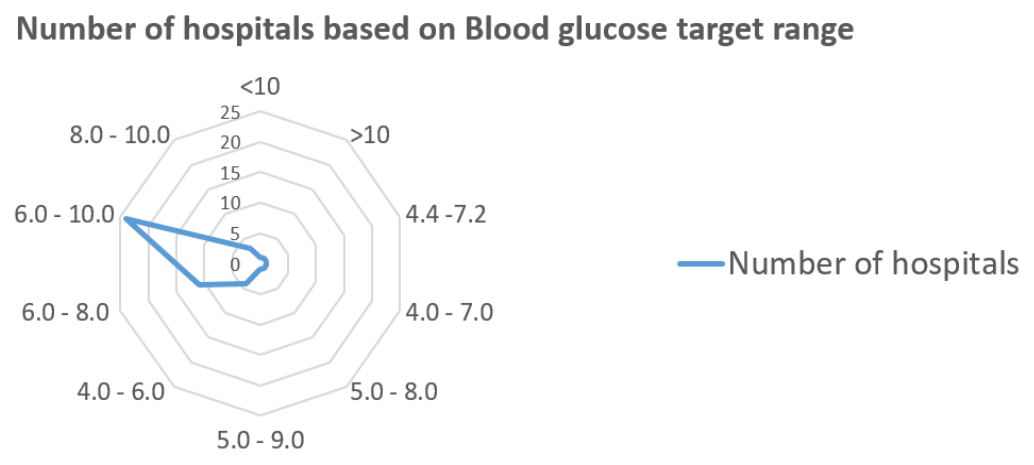

Figure 1. The composition chart of the number of hospitals in Malaysia practicing different glycemic control target range

The main aim of this study was to extend the advantages of the SI and STAR virtual trial framework $[18,19]$ to Malaysian patients, based on assessments of the safety and performance of different target ranges. Second, we used the STAR virtual trial to identify the favorable BG target range for glycemic control in critically ill patients in a Malaysian cohort.

\section{METHODS}

\subsection{Study Design}

Clinical data of ICU patients from two hospitals were used for this study. These were the International Islamic University Malaysia Medical Centre (IIUM) and the Hospital Tengku Ampuan Afzan (HTAA), which are both based in Kuantan, Malaysia. The ethics committees of IIUM and HTAA granted approval for the analysis and publication of these data. Both sets of data for critically ill patients were considered as different cohorts and were then analyzed together and separately using the same virtual patient generation method. Five simulations were performed using the virtual patient data, using STAR protocol, and with the following target ranges: 4.4-8.0, 4.0-6.0, 6.0-8.0, 6.0-10.0, and 8.0-10.0 mmol/L. 


\subsection{Physiological Model and Virtual Patients}

The model used to create virtual patients was the clinically validated Intensive Control InsulinNutrition-Glucose (ICING) model [14, 15]. This model consists of seven mathematical equations to determine the following: BG level, interstitial insulin level, plasma insulin level, gut and stomach glucose levels, enteral glucose input, and endogenous insulin production. Details of this model and its equations are available in [19]. With the exception of model-based insulin sensitivity, which was measured hourly using the integral-based fitting method [20], all parameters were identified and finalized based on the data obtained from other research studies. SI is representative of the underlying metabolic condition of the whole body, capturing patient-specific deviation from model population parameters. A previous study has shown to be relatively independent of both exogenous insulin and exogenous nutrition, allowing it to be used to compute the likely BG response to treatments other than those administered clinically [18]. To generate virtual patients, clinical data, comprising of BG measurements and insulin and/or nutrition inputs, is used with mathematical model to identify a model-based profile for each patient. These profiles, are addressed as virtual patients and can be used to simulate the response to a new or modified set of insulin and/or nutrition inputs [21], and using any type of protocol to generate simulated blood glucose outcomes.

\subsection{Analysis by Virtual Simulation}

Different glycemic control protocols can be tested, virtually assessing likely BG outcomes in individual patients and across a cohort in a simulation. BG outcomes are a function of , its variability, and the protocol that normally includes the chosen BG target range. Dickson et al. [19] have proven that this method is generalizable to any patient and with respect to several protocols. The virtual patient and virtual simulation framework are illustrated in Figure 2. If the virtual trial is generalizable, a proposed simulationbased analysis comparing outcomes for different target ranges with the STAR protocol might give the favorable target ranges. Five target ranges are used in this analysis (4.0-6.0, 4.4-8.0, 6.0-8.0, 6.0-10.0, and $8.0-10.0 \mathrm{mmol} / \mathrm{L}$ ). The $4.4-8.0 \mathrm{mmol} / \mathrm{L}$ range is the default target range for the STAR protocol, and the other four are the target ranges used by $88 \%$ of Malaysian governmental hospitals. While the $4.4-8.0 \mathrm{mmol} / \mathrm{L}$ range is not used with any patient in Malaysia, this target range was included to allow comparison with the Christchurch, Gyulà, and Liege cohorts [17], [22-24].

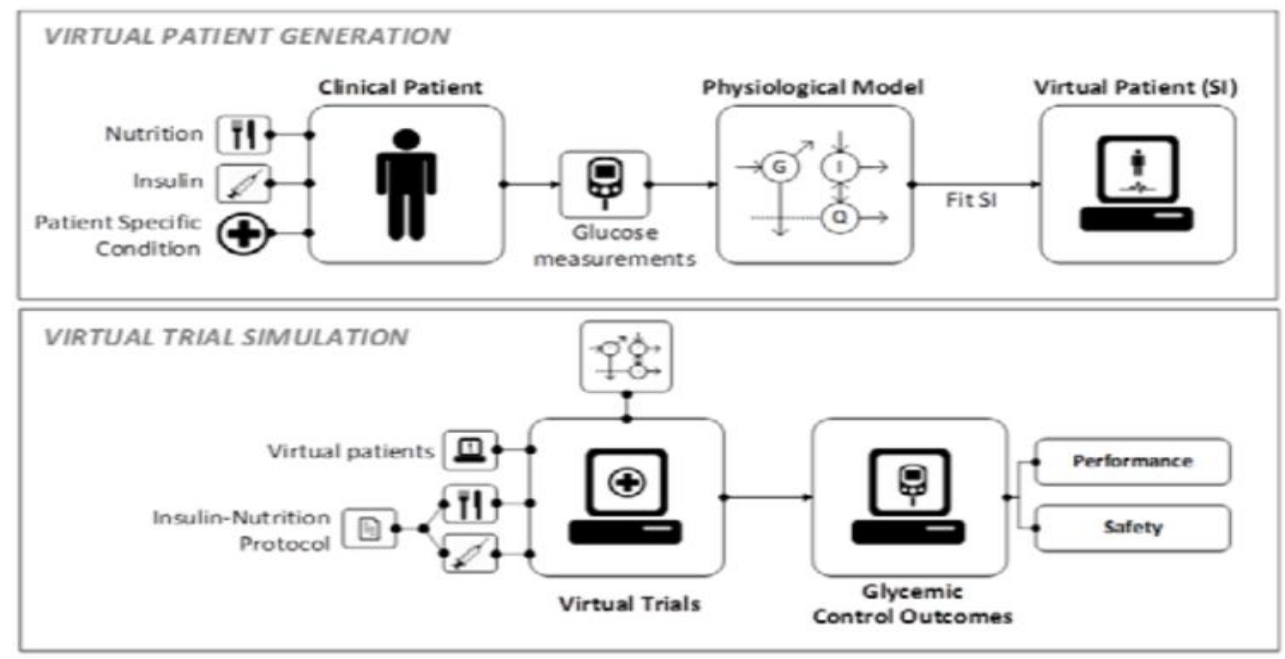

Figure 2. Virtual patient generation and virtual simulation framework. The image is retrieved from [19]

The results are presented as percentage of time BG measurements are at certain BG levels: for example, a BG level of $>10.0 \mathrm{mmol} / \mathrm{L}$ (limit of hyperglycemia), BG within the normoglycemia range, a BG level between 6-10 mmol/L, a BG level of 4.4-8.0 mmol/L, a BG level of $<4.0 \mathrm{mmol} / \mathrm{L}$ (limit of mild hypoglycemia), and a BG level of $<2.2 \mathrm{mmol} / \mathrm{L}$ (limit of severe hypoglycemia). The Mann-Whitney ranksum test was then used to compare the results from the two cohorts. This non-parametric statistic was chosen because of the typically skewed distributions of BG, insulin doses, and other data. $\mathrm{P}$ values of $<0.05$ were considered statistically significant.

The virtual trial can also be used to perform self-simulation to test for model errors or any unmodeled non-compliance to a clinical implementation. Conceptually, testing a virtual patient on the same 
protocol that clinically produced the data should return the same BG data used to generate it, but with differences due to fitting error or in clinically following the protocol recommendation. Self-simulation was performed in the cohort that underwent the STAR pilot trial.

\subsection{Patient Data and Applied Insulin Therapy}

IIUM patients treated using the STAR protocol were recruited from January 1 to November 5, 2017. The data comprised 27 episodes of 16 patients with a minimum of $24 \mathrm{~h}$ of control using the STAR protocol. This protocol recommended both insulin and nutrition doses, requiring hourly or three-hourly measurements of BG when outside or within the set target range, respectively. The starting criterion was two BG measurements of $>10 \mathrm{mmol} / \mathrm{L}$ within a 1 hour period, and the goal was to control BG level within 6.0-10.0 $\mathrm{mmol} / \mathrm{L}$ with a $5 \%$ risk of $\mathrm{BG}$ level of $<4.0 \mathrm{mmol} / \mathrm{L}(72 \mathrm{mg} / \mathrm{dL})$ for each intervention. Contrary to availability of nutrition recommendation in the STAR control, IIUM administered intravenous-infusion insulin only therapy. When clinical opinion has determined that a patient is stable and insulin treatment should end or more than 5 hours has elapsed between treatment, STAR was stopped. Additionally, in this ICU, the stopping criteria was when the BG is within target range at insulin of $0.5 \mathrm{U} / \mathrm{hr}$ for two hours. A comparison of the clinical results with a simulated $6.0-10.0 \mathrm{mmol} / \mathrm{L}$ will present the self-simulation analysis.

A total of 210 patient data with episodes for a minimum of $24 \mathrm{~h}$ were included in the second cohort from HTAA. The standard of glycemic control was based on the sliding scale method, dosing insulin based on the patient's BG level and previous insulin dose, with the final target range of 4.0-6.0 mmol/L. The insulin rate was adjusted based on the current and previous BG level and the current insulin infusion rate, and the intervention was started when patients reached a BG level of at least $10 \mathrm{mmol} / \mathrm{L}$ for about 1 hour. BG was initially monitored hourly until it was within the target range for 2 hour and was then monitored every 4 hour. Soluble insulin was used for continuous intravenous insulin infusion. This approach to control was limited by the inability to reduce the insulin infusion rates until BG was within the target range [25].

Both cohorts were based in Kuantan, Malaysia. Enteral and parenteral nutrition were used in both ICUs, although enteral therapy alone was used in most cases. Ultimately, the decision was made by the clinical staff, but the guideline recommends a target energy intake of $25 \mathrm{kcal} / \mathrm{kg} / \mathrm{day}$ and at least $1.2-1.5$ $\mathrm{g} / \mathrm{kg} / \mathrm{day}$ of protein, adjusting the energy intake as per the severity and type of illness.

\section{RESULTS}

\subsection{IIUM Results}

The results for IIUM cohort is summarized by whole cohort statistics in Table 1 . The median and IQR nutrition rates reported in the table are distinguished per clinical and simulated target ranges results. STAR clinical outcomes using 1-3 hourly interventions averaging 1 measurement every $\sim 1.27$ hours. For virtual simulations, the average ranges from 1 measurement every $\sim 0.99$ to 1.44 hours. If we focus on the self-simulation cohort of $6.0-10.0 \mathrm{mmol} / \mathrm{L}$, the difference of averaged 0.17 hours with clinical trial is largely gained through the number of BG measurements instead of the total hours on therapy. This result hints the non-compliance on nurses' intervention on insulin treatment recommendation. Nurses seemed to introduce more insulins as compared to what were recommended.

The median insulin rate in the clinical trial was $1.7 \mathrm{U} / \mathrm{h}$ and the median dextrose rate was $5.4 \mathrm{~g} / \mathrm{h}$. In the simulation cohorts, the highest insulin rate was in the $4.0-6.0 \mathrm{mmol} / \mathrm{L}$ target range $(5.5 \mathrm{U} / \mathrm{h})$, although the dextrose rate was the lowest $(1.9 \mathrm{~g} / \mathrm{h})$. Surprisingly, there was a $0.0 \mathrm{U} / \mathrm{h}$ median insulin rate in the $8.0-10.0$ $\mathrm{mmol} / \mathrm{L}$ simulation, whereas the dextrose rate was higher $(4.9 \mathrm{~g} / \mathrm{h})$ and closest to that in the clinical trials. Except for the $8.0-10.0 \mathrm{mmol} / \mathrm{L}$ simulation, the maximum per-patient insulin rate was consistent across cohorts (median $8.0 \mathrm{U} / \mathrm{h}$ ). Thus, the STAR protocol is flexible enough to control ranges of insulin and dextrose doses based on a patient's SI and the therapeutic target, while considering safety and daily caloric requirements.

Notably, in the BG level performances, clinical trial resulted with the second highest BG mean after the $8.0-10.0 \mathrm{mmol} / \mathrm{L}$ simulation with $8.6 \%$. Between the simulations, $4.0-6.0$ range scores the lowest $\mathrm{BG}$ mean $(6.2 \%)$, and $6.0-10.0 \mathrm{mmol} / \mathrm{L}$ range recorded a lower $\mathrm{BG}$ mean than clinical's, with $7.80 \mathrm{mmol} / \mathrm{L}$. In term of performance in given targeted ranges, for $6.0-10.0 \mathrm{mmol} / \mathrm{L}$, the clinical trial resulted in $62.44 \%$ and its simulation is higher at 74.86\%. 4.4-8.0, 4.0 -6.0, 6.0-8.0 and 8.0-10.0 mmol/L each performed with $67.65 \%, 38.77 \%, 71.61 \%$ and $46.61 \%$ respectively. Further analysis was done on the performance over hyper- and hypoglycemia limits. The lowest and tighter target range of 4.0-6.0 derived the lowest percentage of hyperglycemia (9.93\%) while the highest naturally came from the highest target range of 8.0-10.0 (28.25 $\%)$. For mild hypoglycemia, STAR clinical and simulations assured the less than 5\% limit, except for the 4.0-6.0 simulation (5.87\%). Severe hypoglycemia percentages ranges from 0.07 to 0.14 
Table 1. IIUM and HTAA Cohorts Glycemic Control Results

\begin{tabular}{|c|c|c|c|c|c|c|}
\hline \multirow{2}{*}{$\begin{array}{l}\text { Performance and safety } \\
\text { statistics for } \\
\text { IIUM cohort }\end{array}$} & \multirow[b]{2}{*}{ Clinical trial } & \multicolumn{5}{|c|}{ STAR virtual simulations } \\
\hline & & $4.4-8.0$ & $4.0-6.0$ & $6.0-8.0$ & $6.0-10.0$ & $8.0-10.0$ \\
\hline No. of patients (Male) & $\underline{16(11)}$ & & & & & \\
\hline No. of episodes & $\underline{27}$ & & & & & \\
\hline Number of BG measurements & $\overline{1070}$ & 998 & 1148 & 1362 & $\underline{943}$ & 1206 \\
\hline Total no. of hours & $\overline{1361}$ & $\overline{1358}$ & $\overline{1353}$ & $\overline{1343}$ & $\overline{1357}$ & $\overline{1350}$ \\
\hline Average measurement interval & $\overline{1.27}$ & $\overline{1.36}$ & $\overline{1.18}$ & $\overline{0.99}$ & 1.44 & 1.33 \\
\hline $\begin{array}{l}\text { Insulin rate }(\mathrm{U} / \mathrm{hr}): \text { Median } \\
{[\mathrm{IQR}]}\end{array}$ & $\frac{\overline{1.7[0.4-}}{3.4]}$ & $\overline{4.0}[2.0-6.5]$ & $\underline{5.5[2.0-8.0]}$ & $\overline{2.0[0.0-4.0]}$ & $\overline{2.0[0.0-3.5]}$ & $\overline{0.0}[0.0-1.0$ \\
\hline $\begin{array}{l}\text { Max Insulin rate (U/hr): } \\
\text { Median [IQR] }\end{array}$ & $\begin{array}{l}\overline{8.0}[5.8- \\
8.0]\end{array}$ & $\underline{8.0[8.8-8.0]}$ & $\underline{8.0[8.0-8.0]}$ & $\underline{8.0[8.0-8.0]}$ & $\underline{8.0[6.3-8.0]}$ & $\underline{6.0[5.3-6.0]}$ \\
\hline $\begin{array}{l}\text { Dextrose rate }(\mathrm{g} / \mathrm{hr}) \text { : Median } \\
\text { [IQR] }\end{array}$ & $\frac{\overline{5.4[2.6-}}{5.9]}$ & $\underline{2.6[0.0-5.2]}$ & $\underline{1.9[0.0-3.9]}$ & $3.9[0.0-6.5]$ & $\underline{3.9[0.0-6.6]}$ & $\underline{4.9[0.0-6.6]}$ \\
\hline Dextrose rate: $(\%$ goal $))$ & $\underline{\underline{\mathrm{NA}}}$ & $\frac{39.7[0.0-}{79.5]}$ & $\frac{29.8[0.0-}{59.6]}$ & $\underline{59.6[0.0-6.5]}$ & $\frac{59.6[0.0-}{100.0]}$ & $\frac{74.5[0.0-}{100.0]}$ \\
\hline Mean BG (mmol/L) & $\underline{8.60}$ & $\overline{6.80}$ & $\overline{6.20}$ & $\underline{7.50}$ & 7.80 & 9.00 \\
\hline$\% \mathrm{BG}>10 \mathrm{mmol} / \mathrm{L}$ & $\overline{28.67}$ & $\overline{11.26}$ & $\overline{9.93}$ & $\overline{14.60}$ & $\overline{15.32}$ & $\overline{28.25}$ \\
\hline \%BG within $4.4-8.0 \mathrm{mmol} / \mathrm{L}$ & 36.25 & 67.65 & 65.80 & 60.00 & 52.24 & 24.47 \\
\hline$\%$ BG within $6.0-10.0 \mathrm{mmol} / \mathrm{L}$ & 62.44 & $\overline{58.41}$ & 45.43 & 71.61 & 74.86 & $\underline{68.43}$ \\
\hline$\overline{\% \mathrm{BG} \text { within } 4.0-10.0 \mathrm{mmol} / \mathrm{L}}$ & $\overline{70.67}$ & $\overline{84.99}$ & $\overline{84.20}$ & $\overline{84.09}$ & $\overline{83.81}$ & $\overline{71.02}$ \\
\hline$\% \mathrm{BG}<4.0 \mathrm{mmol} / \mathrm{L}$ & 0.66 & 3.75 & 5.87 & 1.31 & 0.87 & 0.73 \\
\hline$\% \mathrm{BG}<2.2 \mathrm{mmol} / \mathrm{L}$ & $\underline{0.07}$ & 0.22 & 0.58 & 0.36 & 0.14 & 0.07 \\
\hline Performance and safety & $\overline{\text { Clinical trial }}$ & $\overline{\text { STAR VIRTUA }}$ & $\overline{\text { SIMULATIONS }}$ & & & \\
\hline $\begin{array}{l}\text { statistics for } \\
\text { HTAA cohort }\end{array}$ & $\frac{(\mathrm{SS} 4.0-}{\underline{6.0)}}$ & $\underline{4.4-8.0}$ & $\underline{4.0-6.0}$ & $\underline{6.0-8.0}$ & $\underline{6.0-10.0}$ & $\underline{8.0-10.0}$ \\
\hline No. of patients (Male) & $\overline{210}(119)$ & & & & & \\
\hline No. of episodes & 210 & & & & & \\
\hline$\overline{\text { Number of BG measurements }}$ & 13011 & $\underline{17580}$ & $\underline{21385}$ & 26675 & $\underline{17070}$ & $\underline{22775}$ \\
\hline Total no. of hours & 26727 & 26610 & 26573 & 26518 & 26619 & 26560 \\
\hline Average measurement interval & 2.05 & 1.51 & 1.24 & 0.99 & 1.56 & 1.17 \\
\hline $\begin{array}{l}\text { Insulin rate }(\mathrm{U} / \mathrm{hr}): \text { Median } \\
{[\mathrm{IQR}]}\end{array}$ & $\frac{\overline{2.0}[1.0-}{3.0]}$ & $\overline{5.0[2.0-8.0]}$ & $\overline{6.0[3.0-8.0]}$ & $\overline{2.5[0.0-6.0]}$ & $\overline{2.0[0.0-4.0]}$ & $\overline{0.0}[0.0-2.0]$ \\
\hline $\begin{array}{l}\text { Max Insulin rate (U/hr): } \\
\text { Median [IQR] }\end{array}$ & $\frac{4.0[3.0-}{5.5]}$ & $\underline{8.0[8.0-8.0]}$ & $\underline{8.0[8.0-8.0]}$ & $\underline{8.0[8.0-8.0]}$ & $\underline{8.0[8.0-8.0]}$ & $\underline{6.0[5.5-8.0]}$ \\
\hline $\begin{array}{l}\text { Dextrose rate }(\mathrm{g} / \mathrm{hr}) \text { : Median } \\
\text { IQR] }\end{array}$ & $\frac{4.1[2.6-}{6.0]}$ & $\underline{3.9[1.9-6.5]}$ & $\underline{1.9[1.9-4.5]}$ & $\underline{4.6[1.9-6.6]}$ & $\underline{5.5[1.9-6.6]}$ & $\underline{6.5[3.9-6.6]}$ \\
\hline Dextrose rate: $(\%$ goal $))$ & $\underline{\mathrm{NA}}$ & $\frac{59.6[29.8-}{99.3]}$ & $\frac{29.8[29.8-}{69.5]}$ & $\frac{70.8[29.8-}{100.0]}$ & $\frac{84.4[29.8-}{100.0]}$ & $\frac{100[59.6-}{100.0]}$ \\
\hline Mean BG (mmol/L) & $\underline{8.6}$ & 6.9 & 6.3 & 7.6 & 8.3 & 9.6 \\
\hline$\% \mathrm{BG}>10 \mathrm{mmol} / \mathrm{L}$ & $\underline{31.26}$ & 14.69 & 12.89 & 19.89 & $\overline{23.40}$ & 40.32 \\
\hline 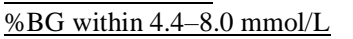 & $\overline{40.62}$ & $\overline{63.16}$ & $\overline{64.94}$ & $\overline{56.05}$ & $\overline{43.76}$ & $\overline{19.14}$ \\
\hline$\%$ BG within $6.0-10.0 \mathrm{mmol} / \mathrm{L}$ & $\underline{56.46}$ & 56.11 & $\underline{43.26}$ & 71.35 & 70.15 & 57.52 \\
\hline$\% \mathrm{BG}$ within $4.0-10.0 \mathrm{mmol} / \mathrm{L}$ & 67.79 & 69.31 & 83.44 & 79.68 & 91.3 & 59.60 \\
\hline$\% \mathrm{BG}<4.0 \mathrm{mmol} / \mathrm{L}$ & 0.95 & 2.16 & 3.67 & 0.43 & $\overline{0.40}$ & 0.08 \\
\hline$\% \mathrm{BG}<2.2 \mathrm{mmol} / \mathrm{L}$ & $\underline{0.04}$ & $\underline{0.06}$ & $\underline{0.11}$ & $\underline{0.02}$ & $\underline{0.01}$ & $\underline{0.00}$ \\
\hline
\end{tabular}

\subsection{HTAA results}

The results for the HTAA cohort are also summarized in Table 1. The cohort was 13 times bigger than the IIUM cohort, but the acquired data (hours and BG measurements) were 20 times bigger. The IIUM cohort tended to have shorter episodes because the STAR protocol led to a quick stabilization of glycemic levels. The clinical trial used an insulin sliding scale therapy and set a BG target range of 4.0-6.0 mmol/L, with the HTAA profiles used to assess the performances of simulations of target ranges compared with the IIUM cohort. In terms of averaged 1 over hours of BG intervention, similar to IIUM virtual simulations, the larger target range of 4.4-8.0 and 6.0-10.0 mmol/L have the higher 1 over average of 1.51 and 1.56 hour measurements. The simulated values when compared with the clinical data ( 1 over 2.05 hours) however shows that STAR protocol tends to introduce more clinical intervention.

The clinical data showed the median insulin rate and per-patient max insulin rate to be similar to the 6.0-8.0 and 6.0 - 10.0 simulations but half of those simulated using lower target ranges simulation (4.0-6.0 and 4.4-8.0). The median dextrose rates are however vary without specific pattern. Similarly to the IIUM cohort analysis, Simulation from target range of 4.0-6.0 $\mathrm{mmol} / \mathrm{L}$ introduced the highest median insulin rate and simulation 8.0-10.0 introduced the highest dextrose rate. Per-patient maximum insulin rate records the same consistency at 8.0 and $6.0 \mathrm{U} / \mathrm{hr}$. The glycemic control protocol applied in HTAA organizes the nutrition therapy separated from insulin infusion, it may introduces differences to the variations if compared to the IIUM's cohort. However from the similarities of results thus far, positively a comparison of BG performance can be done with the IIUM results. 
To consolidate our findings, the Mann-Whitney test was conducted for the comparison of the IIUM and HTAA cohorts (Table 2). The analysis was based on both the original protocol and the virtual simulation using the representative target range of $6.0-10.0 \mathrm{mmol} / \mathrm{L}$. The Mann-Whitney test was used to assess the two-tailed distribution of significant differences between the two cohorts. None of the variables, including the mean BG performance, were significantly different. The mean BG level for the original protocol and virtual simulation produced a P-value of 0.051 , which is close to the significance threshold.

Table 2. Comparison of the IIUM and HTAA Cohorts at the $6.0-10.0 \mathrm{mmol} / \mathrm{L}$ Target Range using the Mann-Whitney test

\begin{tabular}{lccc}
\hline $\begin{array}{l}\text { Performance and safety statistics due to } \\
\text { 6.0-10.0 mmol/L target range }\end{array}$ & IIUM cohort & HTAA cohort & Mann Whitney test \\
\hline Original protocol & $1.7[0.4-3.4]$ & $2.0[1.0-3.0]$ & P: 0.80 \\
Insulin rate (U/hr): median [IQR] & $5.4[2.6-5.9]$ & $4.1[2.6-6.0]$ & P: 0.90 \\
Dextrose rate (g/hr): Median [IQR] & 8.76 & 8.58 & P: 0.14 \\
Mean BG (mmol/L) & 8.60 & 8.60 & P: 0.05 \\
Mean BG (mmol/L) & & & \\
Virtual simulation & $2.0[0.0-3.5]$ & $2.0[0.0-4.0]$ & P: 0.80 \\
Insulin rate (U/hr): median [IQR] & $3.9[0.0-6.6]$ & $5.5[1.9-6.6]$ & P: 0.906 \\
Dextrose rate (g/hr): Median [IQR] & 7.97 & 8.39 & P: 0.14 \\
Mean BG (mmol/L) & 7.80 & 8.30 & P: 0.05 \\
Mean BG (mmol/L) & &
\end{tabular}

In terms of BG performance, the clinical trials and simulations produced similar mean BG values. Between the simulations, the $4.0-6.0 \mathrm{mmol} / \mathrm{L}$ range produced the lowest mean $\mathrm{BG}$. Performance in given targeted ranges was the highest for the $6.0-10.0 \mathrm{mmol} / \mathrm{L}$ at $70.15 \%$, followed by the $4.4-8.0 \mathrm{mmol} / \mathrm{L}$ simulation at $63.16 \%$. The other simulations had performances of $<50 \%$, presumably because they used stricter target ranges. The performance values inside the normoglycemic range $(4.4-10.0 \mathrm{mmol} / \mathrm{L}) \mathrm{varied}$ from $59.60 \%$ to $83.44 \%$. The simulation records in the HTAA cohort for $4.0-6.0 \mathrm{mmol} / \mathrm{L}$ had the lowest mean value of $6.30 \%$ and the lowest percentage of $\mathrm{BG}$ of $>10 \mathrm{mmol} / \mathrm{L}$, but also had the highest number of patients with severe hypoglycemia $(0.11 \%)$. Indeed, the percentage of patients with severe hypoglycemia was more than double that in the clinical trial $(0.04 \%)$, which shared the same target range, but used a sliding scale. The results indicated that all ranges were safe ( $<5 \%$ with mild hypoglycemia). Moreover, the $8.0-10.0$ $\mathrm{mmol} / \mathrm{L}$ simulation resulted in no cases of severe hypoglycemia in the HTAA cohort and very few cases in the IIUM cohort $(0.07 \%)$.

\section{DISCUSSION}

To present the performance of each target range simulation, the results from the cohort analysis are summarized in Figure 3. The primary y-axis shows the performance for each range (different colored bars) with the percentages of hyperglycemia (red lines). The secondary y-axis shows the percentages of hypoglycemia at the two limits (black lines and dashes). The normoglycemia range is also displayed in the primary $y$-axis (green bars). To highlight the performance per simulation according to the given target range, the colored bars are denoted with a line pattern. Comparing the cohorts, two conclusions can be drawn. First, if the STAR protocol is used with $100 \%$ compliance, performance can be improved using the proposed 6.0$10.0 \mathrm{mmol} / \mathrm{L}$ target range. This is consistent with the range used in the pilot trial at IIUM, and it benefits from minimizing the risks of hyperglycemia and hypoglycemia. It also provided the highest simulated performance in both the IIUM and HTAA cohorts.

Second, virtual simulations in the Malaysian patients showed that the $6.0-8.0$ and $6.0-10.0 \mathrm{mmol} / \mathrm{L}$ ranges optimized the rate of hyperglycemic and hypoglycemic events. While the performance of the 6.0-8.0 $\mathrm{mmol} / \mathrm{L}$ simulation was low, the time its patients spend within the $4.4-10.0 \mathrm{mmol} / \mathrm{L}$ range was comparable to that within the $6.0-10.0 \mathrm{mmol} / \mathrm{L}$ simulation. To decide the better option, a comparison was done using the introduced insulin, introduced dextrose, targeted dextrose rate achieved, and measurement intervals. The first three variables gave similar values, and only the measurement interval produced a difference. The 6.0-10.0 $\mathrm{mmol} / \mathrm{L}$ simulation averaged 1.44 and 1.56 number of measurements every hour in the IIUM and HTAA cohorts, respectively, indicating fewer interventions, thus less workloads.

Focusing solely on the performance of the 4.4-8.0 $\mathrm{mmol} / \mathrm{L}$ target range in the IIUM and HTAA cohorts hinted at the ability of the STAR protocol to achieve glycemic control with acceptable rate of hyperglycemic and hypoglycemic events. However, their respective simulated values of $67.65 \%$ and $63.16 \%$ were very low compared with the virtual trial results of Dickson et al. [19], who reported a minimum of 
$81.0 \%$ of time spent in the $4.4-8.0 \mathrm{mmol} / \mathrm{L}$ target range among patients in the Gyulà and New Zealand cohorts. The result is, however, similar to that in the Belgian report of patients who underwent insulin control with the sliding scale method. This finding supports the hypothesis that Malaysian cohorts have different rate of metabolism that requires them to have different target ranges to achieve optimal glycemic control, both in general and when using the STAR protocol. Although this analysis was only statistical, we conclude that Malaysian patients will gain most benefit from the target range of $6.0-10.0 \mathrm{mmol} / \mathrm{L}$ in terms of controlling hyperglycemia, preventing hypoglycemia, and reduced workload. Currently, only $50 \%$ of government and research university hospitals use this target range, which we believe should be used for future STAR trials in these hospitals.

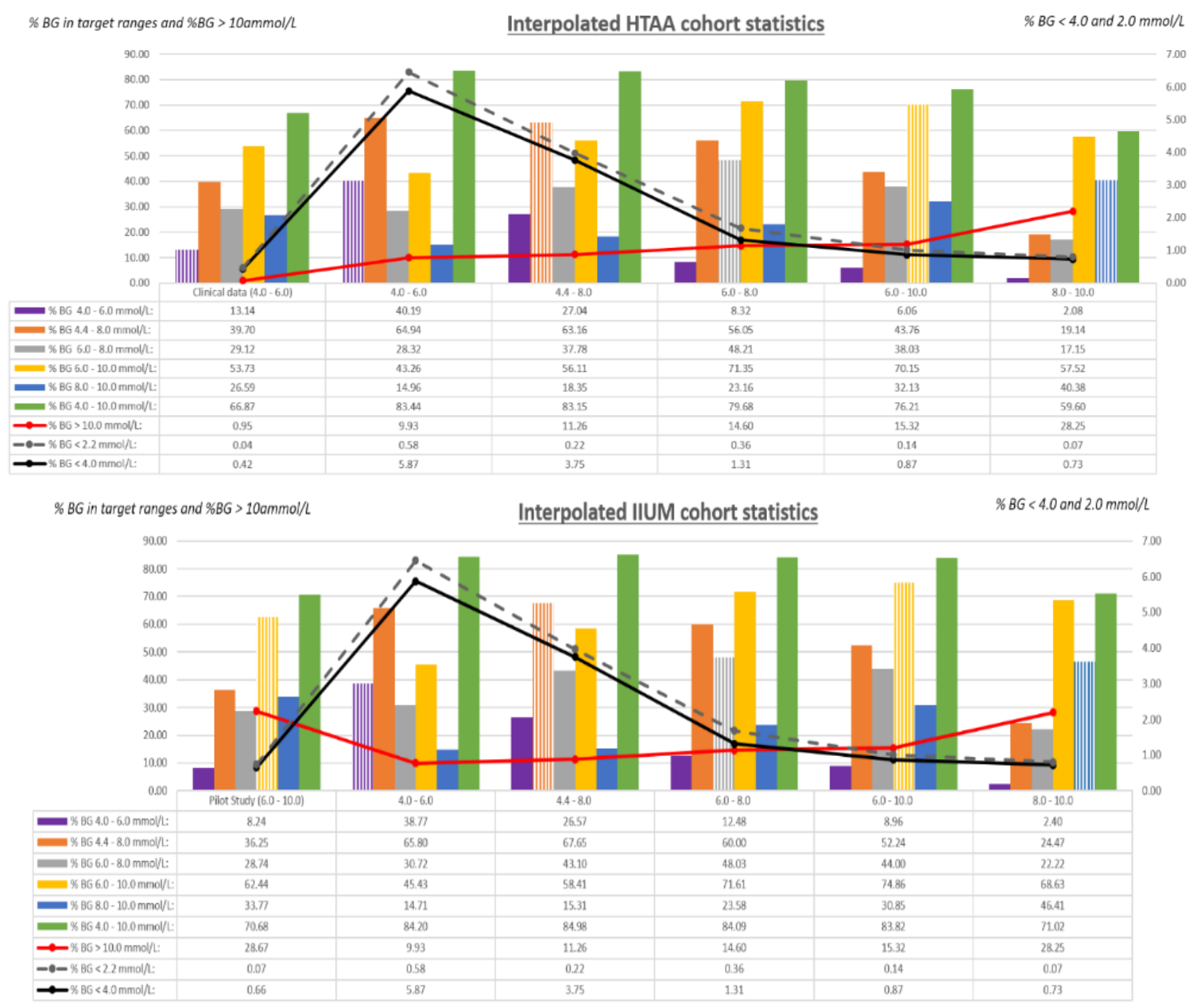

Figure 3. Interpolated IIUM and HTAA cohort statistics

Finally, a limitation of our simulations was that they relied on virtual patients drawn from two ICUs from the same region in Malaysia. These patients may different underlying metabolisms from other Malaysian patients, which would have affected the generalizability to all Malaysians. However, this study provides relevant data and should prompt further investigation into these demographic factors, such as ethnicity, which may affect the performance of the STAR protocol in Indonesia, India, and China, as Malaysian are generally constituted of Malays, Indian and Chinese.

\section{CONCLUSION}

In this research, we have presented an analysis of virtual patients from two different ICUs using a model-based virtual trial framework in which BG target ranges were varied and compared with each other. Patients were included to test the performance and safety of the STAR framework in Malaysian patients, seeking to extend its generalizability and to identify the favorable target range. We showed that the non-

Determination of favorable blood glucose target range for stochastic TARgeted (STAR)... (A. Abu-Samah) 
default STAR target range of $6.0-10.0 \mathrm{mmol} / \mathrm{L}$ had the best balance of performance, safety, and frequency of nursing interventions among the target ranges tested. This result is encouraging as it is consistent with the target range recommended for achieving glycemic control in other scenarios with respect to Malaysian patients.

\section{ACKNOWLEDGMENT}

Written informed consent was obtained for all patients, and approval was granted for this study by the institutional research ethics committees of IIUM (IREC 657) and HTAA (NMR13-1592-18706), as well as by the National Institute of Health (NIH). The authors acknowledge clinical teams of the ICUs at IIUM and HTAA for their support and provision of data for this study. The authors also acknowledge the FRGS grant from Ministry of Higher Education, the BOLD Scheme, and UNITEN for their roles with the ongoing research.

\section{REFERENCES}

[1] G. Van Den Berghe, et al., "Intensive Insulin Therapy in Critically Ill Patients," New England Journal of Medicine, vol. 345, no. 19, pp. 1359-1367, 2001.

[2] G. Van den Berghe, et al., "Intensive Insulin Therapy in the Medical ICU," New England Journal of Medicine, vol. 354, no. 5, pp. 449-461, 2006.

[3] Y. M. Arabi, et al., "Intensive versus Conventional Insulin Therapy: a Randomized Controlled Trial in Medical and Surgical Critically Ill Patients," Critical Care Medicine, vol. 36, no.12, pp. 3190-3197, 2008.

[4] N.-S. S. Investigators, "Intensive versus Conventional Glucose Control in Critically Ill Patients," New England Journal of Medicine, vol. 360, no. 13, pp.1283-1297, 2009.

[5] A. Evans, et al., "Pilot Proof of Concept Clinical Trials of Stochastic TARgeted (STAR) Glycemic Control," Annals of Intensive Care, vol. 1, no. 1, pp. 38, 2011.

[6] L. L. M. Fisk, et al., "Star Development and Protocol Comparison," IEEE Transactions on Biomedical Engineering, vol. 59, no.12, pp.3357-3364, 2012.

[7] N. M. Saur, et al., "Software-guided Insulin Dosing: Tight Glycemic Control and Decreased Glycemic Derangements in Critically Ill Patients," in: Mayo Clinic Proceedings, vol. 88, pp. 920-929, Elsevier 2013.

[8] S. Cochran, et al., "EndotoolTM Software for Tight Glucose Control for Critically Ill Patients: 260," Critical Care Medicine, vol. 34, no. 12, A68, 2006.

[9] T. Van Herpe, et al., "Logic-Insulin Algorithm-guided versus Nurse-directed Blood Glucose Control during Critical Illness: the Logic-1 Single-center, Randomized, Controlled Clinical Trial,” Diabetes Care, vol. 36, no. (2), pp. 188194, 2013.

[10] A. Evans, et al., "Pilot Proof of Concept Clinical Trials of Stochastic Targeted (STAR) Glycemic Control," Annals of Intensive Care, 1(1):38, 2011.

[11] A. Evans, et al., "Stochastic Targeted (STAR) Glycemic Control: Design, Safety, and Performance," Journal of Diabetes Science and Technology, 6(1):102-115, 2012.

[12] J. Blaha, et al., "Space GlucoseControl System for Blood Glucose Control in Intensive Care Patients- a European Multicentre Observational Study," BMC anesthesiology, 16(1), 8, 2015.

[13] B. Xu, et al., "Comparison of Space Glucose Control and Routine Glucose Management Protocol for Glycemic Control in Critically Ill Patients: A Prospective, Randomized Clinical Study," Chinese Medical Journal, 130(17), $2041,2017$.

[14] J. Lin, et al., "Stochastic Modelling of Insulin Sensitivity and Adaptive Glycemic Control for Critical Care," Computer Methods and Programs in Biomedicine, 89(2), pp.141-152, 2008.

[15] J. Lin, et al., "A physiological Intensive Control Insulin-Nutrition-Glucose (icing) Model Validated in Critically Ill Patients," Computer Methods and Programs in Biomedicine, vol. 102, no. 2, pp.192-205, 2011.

[16] A. Abu-Samah, et al., "Model-based Insulin-Nutrition Administration for Glycemic Control in Malaysian Critical Care: First Pilot Trial," in: International Conference for Innovation in Biomedical Engineering and Life Sciences, pp. 189-196, Springer Dec 2017.

[17] K. W. Stewart, et al., "Safety, Efficacy and Clinical Generalization of the STAR Protocol: a Retrospective Analysis," Annals of Intensive Care, vol. 6, no. 1, pp. 24, 2016.

[18] J. G. Chase, et al., "Validation of a Model-based Virtual Trials Method for Tight Glycemic Control in Intensive Care," Biomedical Engineering Online, Vol. 9, no. 1, 2010.

[19] J. L. Dickson, et al., "Generalisability of a Virtual Trials Method for Glycaemic Control in Intensive Care," IEEE Transactions on Biomedical Engineering, 2017.

[20] C. E. Hann, et al., "Integral-based Parameter Identification for Long-term Dynamic Verification of a GlucoseInsulin System Model," Computer Methods and Programs in Biomedicine, vol. 77, no. 3, pp. 259-270, 2005.

[21] K. W. Stewart, et al., "Stochastic Model Predictive (STOMP) Glycaemic Control for the Intensive Care Unit: Development and Virtual Trial Validation," Biomedical Signal Processing and Control, vol. 16, pp. 61-67, 2015.

[22] S. Penning, et al., "First Pilot Trial of the STAR-Liege Protocol for Tight Glycemic Control in Critically Ill Patients," Computer Methods and Programs in Biomedicine, 108(2), pp.844-859, 2012. 
[23] S. Penning, S., et al., "Second Pilot Trials of the STAR-Liege Protocol for Tight Glycemic Control in Critically Ill Patients," Biomedical Engineering Online, 11(1), p.58, 2012.

[24] V. Uyttendaele, et al., "Preliminary Results from the STAR-Liège Clinical Trial: Virtual Trials, Safety, Performance, and Compliance Analysis," IFAC-PapersOnLine, 51(27), pp.355-360, 2018.

[25] H. Luqman, et al., "Performance of Blood Glucose Management in HTAA Intensive Care Unit," in: Automatic Control and Intelligent Systems (I2CACIS), IEEE International Conference on, IEEE, pp. 156-161, 2016.

\section{BIOGRAPHIES OF AUTHORS}
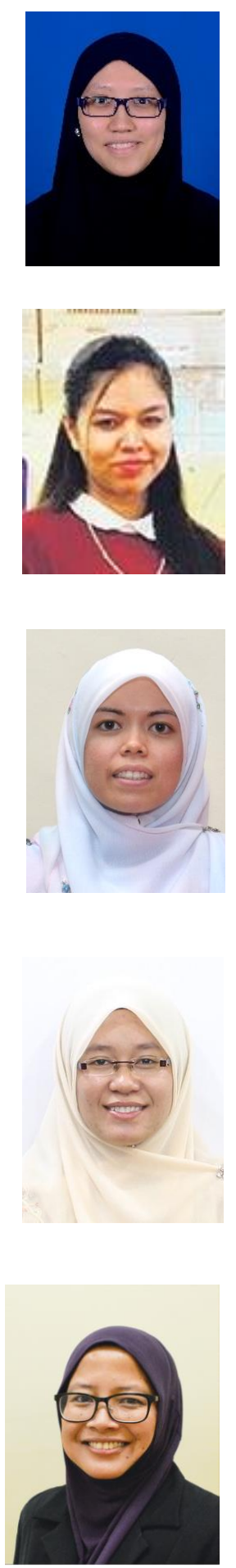

Asma' Abu-Samah is Post-doctoral researcher of Automated Control, applied to Bio-Medical Engineering at the National Energy University (UNITEN), Malaysia. She received her B.Eng and M.Eng. Degrees in Control System of Electrical Energy from Université de Joseph Fourier, France, in 2008 and 2010, respectively, and her Ph.D. in Automated Control and Production Systems from the Université Grenoble Alpes in 2016. Dr. Asma' served or currently serving as a reviewer for many Journals, Conferences, Symposiums, Workshop in Bio-Medical as well as Prognostics and Health Management area. Her research interests include data-based failure prediction and diagnosis in industrial equipment as well as human's physiological system.

Normy Norfiza Abdul Razak is a senior lecturer with the Department of Electronics \& Communication Engineering, National Energy University (UNITEN), Malaysia. She received her B.Eng in Electrical Electronics from UNITEN and M.Sc. Degree in Electrical EngineeringControl and Automation from University of Nottigham, in 2003 and 2014, respectively. She completed her Ph.D. in Bio-Engineering- Computational Modeling and Physiological Control from the University of Canterbury in 2011. Dr. Normy is currently the director of UNITEN Research Management Centre.

Ummu Kulthum Jamaludin is a senior lecturer with the Faculty of Engineering, Universiti Malaysia Pahang, Malaysia. She received her B.Eng with Honours in Mechanical Engineering from University of Canterbury in 2008. This followed by Ph.D. from the same university in 2013. Her expert area includes; System modeling and dynamics, Instrumentation for Biomedical engieering and Physiological modelling.

Fatanah Mohamad Suhaimi is a senior lecturer at the Advanced Medical and Dental Institute (AMDI) of Universiti Sains Malaysia (USM). She received B.Eng in Mechatronics Engineering (Honors) from the International Islamic University Malaysia. In 2012, she was awarded Doctor of Philosophy in Mechanical Engineering from the University of Canterbury, New Zealand. Her research area includes computational modelling of a glucose-insulin system, study and design in bioengineering field specifically in medical instrumentation, control, prediction analysis, and rehabilitation engineering.

Azrina Md Ralib Dr Azrina is an Associate Professor at the Department of Anaesthesiology of IIUM. She is also a full time practicing Consultant Anaesthesiologist at the IIUM Medical Centre, Kuantan. She obtained her Bachelor of Medical Science degree from the University of St. Andrews, UK and Bachelor of Medicine and Bachelor of Surgery (MBChB) degree from the University of Manchester, UK. Upon completion of her bachelor degrees, she returned to Malaysia for her housemanship training. She then joined the Department of Anaesthesiology and Intensive Care in 2002 as trainee lecturer, and started her anaesthesiology training at Universiti Sains Malaysia, Kubang Kerian. She graduated as Clinical Specialist in Anaesthesiology in 2006 with several awards to her name. 